\title{
Residential Energy Resource Models for Distribution Feeder Simulation
}

\author{
R. T. Guttromson, Member, IEEE, D. P. Chassin, and S. E. Widergren, Senior Member, IEEE
}

\begin{abstract}
Advances in information technology, ubiquitous communications, and distributed generation and storage reveal new opportunities for the participation of demand-side resources in balancing the physical and economic operation of electric power systems. To better understand the potential impact of this participation, accurate, detailed energy resource models are necessary at the distribution feeder level. This presentation describes a detailed approach to residential energy resource modeling that preserves the individual characteristics of major residential appliances and human behavior patterns so that their contribution to energy efficiency schemes and intelligent demand curtailment algorithms is properly portrayed. These models are derived from previous analyses of residential and commercial building systems supported by data collected from the End-Use Load and Consumer Assessment Program (ELCAP) undertaken by the Bonneville Power Administration from 1983 to 1990. Preliminary results of using these models in distribution system simulations indicate that non-obvious, complex behavior patterns can emerge when consumers are confronted with varying price signals.
\end{abstract}

Index Terms-adaptive systems, complexity theory, home appliances, load modeling, power distribution, power system simulation

\section{INTRODUCTION}

A $s$ the restructuring of the electric power industry moves from wholesale to retail markets, new incentives are emerging to encourage the participation of demand-side resources in the economic operation of the system. Energy service providers are at various stages of development to incorporate the rising penetration of distributed energy resources in their service areas into their operations portfolio. These resources include distributed generation, storage, and controllable load. Though generally regarded in the experimental phase today, several organizations have programs for commercial sector distributed energy resources to participate in day-ahead energy, emergency energy, and similar markets [1]. Other programs offer time of date rates to commercial or residential customers. The decrease in cost and increase in intelligence of sensors and controllers together

This work is supported by the Pacific Northwest National Laboratory operated for the U.S. Department of Energy by Battelle under Contract DEAC06-76RL01830.

R. T. Guttromson, D. P. Chassin, and S. E. Widergren are with Pacific Northwest National Laboratory, PO Box 999, MSIN: K5-20, Richland, WA 99352 USA (e-mail: ross.guttromson@pnl.gov, david.chassin@pnl.gov, steve.widergren@pnl.gov) with the wide availability of telecommunications over interoperable platforms (wire, fiber, wireless...) is converging with forces in the electric power industry toward higher efficiency, greater utilization of assets, and targeted customer services. Entrepeneurial load serving entities and energy aggregators are moving forward with their creative ideas in this area; however, many questions arise concerning the impacts of such operational changes on the distribution system.

How do these programs affect the diversity of the load profile experienced at the head of the feeder? How far can the peak load be brought down or controlled? What savings could be realized on system upgrades and expansion with such programs? By stimulating active, independent decision making with economic signals, what complex behaviors will a plethora of intelligent devices exhibit?

To address these questions, the empirically driven, noninteractive load models need to give way to sophisticated models that simulate the independent behavior of those components that contribute to the feeder load shape. Specifying a time, date, and temperature for scaling load profiles based on historical data is no longer adequate when energy consumers are given the choice to participate in various programs offered by multiple organizations. To simulate the emergent behavior of potential new economic incentive programs and control schemes requires a significant change in the way demand has been modeled traditionally.

The following sections present the components required for a more detailed modeling of the residential loads and their potential use within a distribution system simulation environment. Though this paper focuses on residential load models, the work represents an initial step forward. Future work is planned to encompass commercial and industrial consumers.

\section{BOTTOM UP APPROACH}

\section{A. Residential Empirical Models}

Nearly all of today's distribution load forecasting is empirically determined. That is, most approaches to determining load growth and load profiles are based on some combination of measurement and probabilities [2], [3].

The contributions from all the load components within the feeder are combined to make a single, lumped model. This top-down form of modeling does not allow the extraction or 
regression of the individual parts from the total load. It is inherently unable to simulate the effects of changes in the interactions between individual load behaviors that contribute to the aggregate load shape at the head of a feeder.

\section{B. Detailed Residential Models}

An alternative to empirical models is a deterministic model. Consider a residential heating ventilation and air conditioning (HVAC) system. By describing this system with differential equations and thermostatic controllers, a computational model for the behavior of the HVAC can be created. Modeling the residential appliance to this level of detail allows the computer to simulate their combined interactions. The result is an aggregated behavior that is not realizable with an empirical model.

An essential objective of the detailed model is to ensure that it accurately reproduces the effects of load diversity. This behavior can be seen in the following graphs of simulations with detailed models of thermostatically controlled appliances (HVAC, water heaters, and refrigerators).
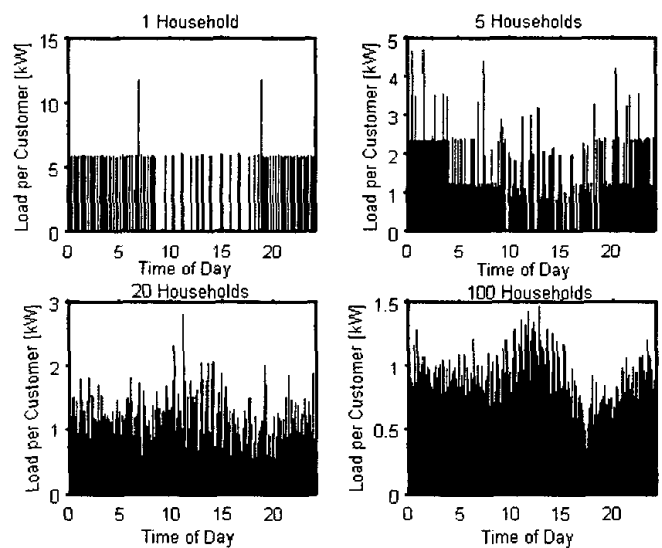

Fig. 1: Load diversity as household loads increase

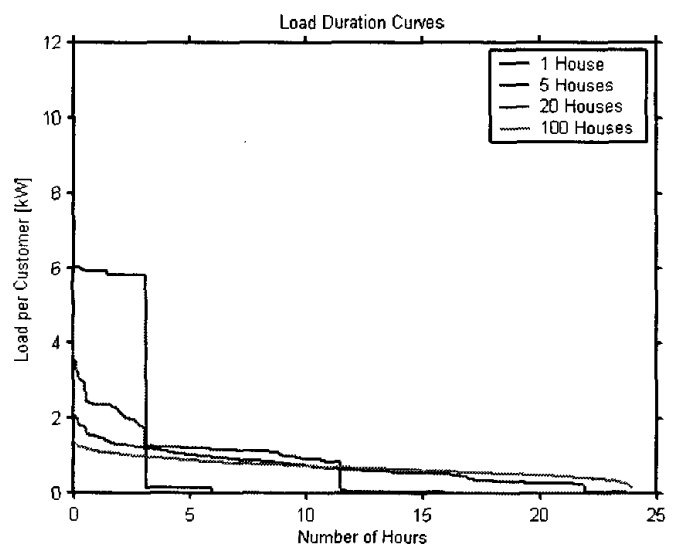

Fig. 2: Load duration curves as diversity increases

Fig. 1 shows that the effects of diversity are indeed represented in the model output. This is verified by looking at the load duration curves in Fig. 2 for the four cases in Fig. 1. As more customers are added to the distribution feeder, the peak load per customer is dropping, which is a direct result of increased diversity.

Combining many similar models, such as HVAC, hot water heater, lighting, and residential appliances, creates a model of a house. Modeling many homes statistically diversifies the model parameters and initial conditions, producing what is in essence a realistic residential feeder model. The model currently used is based on the End-Use Load and Consumer Assessment Program (ELCAP) data collected by the Bonneville Power Administration (BPA) from 1983 to 1990 [4]. From this data we obtained thermal parameters and load shape probability density functions for the most significant building end-uses [5].

When the exogenous inputs vary, such as outside temperature and radiant sunlight, variations on the distribution system are evident. However, if this model is joined with economic market structures (through retail contracts), and the loads are provided with adjustable curtailment algorithms, then a distribution system owner could also use this model to forecast the economic benefit of using alternative rate structures. Such a distribution model is shown in Fig. 3.

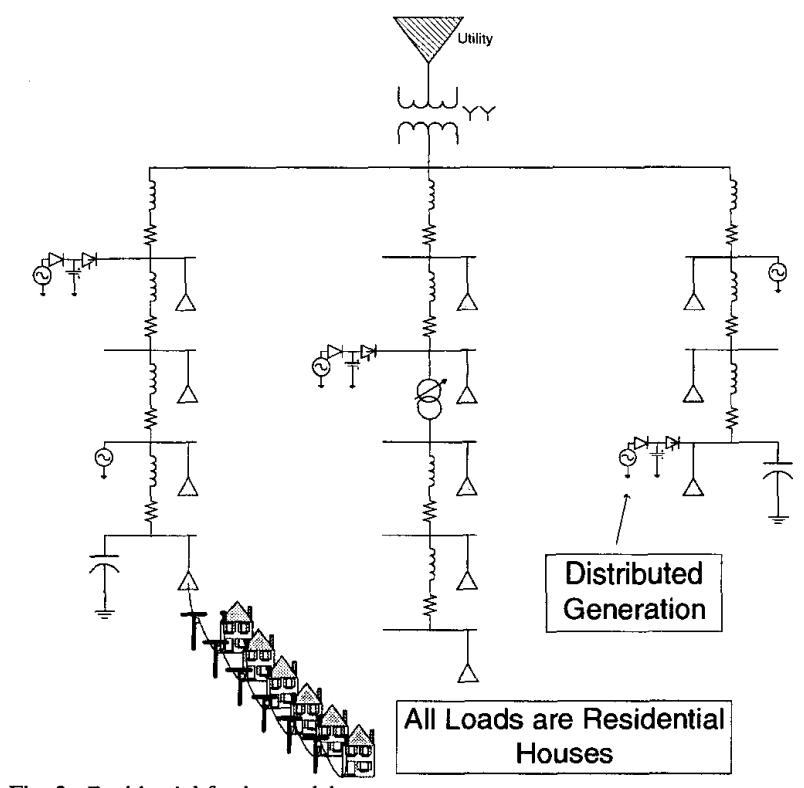

Fig. 3: Residential feeder model

\section{RESIDENTIAL DEMAND TYPES}

Residential appliances can be categorized into those that are thermostatically controlled and those that are nonthermostatically controlled. In addition, the human behavior that controls the setpoints and use of these appliances must also be modeled.

\section{A. Thermostatically Controlled Appliances}

There are three types of thermostatically controlled appliances allowed in the model. They are HVAC systems, electric water heaters, and refrigerators. Each of these 
appliances is modeled in a similar manner, so we shall focus only on the HVAC model for illustrative purposes.

A major assumption integrated into this process is that appliances controlled by a thermostat curtail load by adjusting their thermostat setpoint. For example, if the distribution system reports to the HVAC system that the cost of electricity has just risen from 5 cents per $\mathrm{kWh}$ to 15 cents per $\mathrm{kWh}$, then the HVAC system would respond (within bounds) by lowering the heater's thermostat setpoint, or alternatively, raising the air conditioning setpoint. The entire process of developing a parametric thermal model based on end-use data is called Equivalent Thermal Parameters and is illustrated in Fig. 4, below, see [6]-[8].

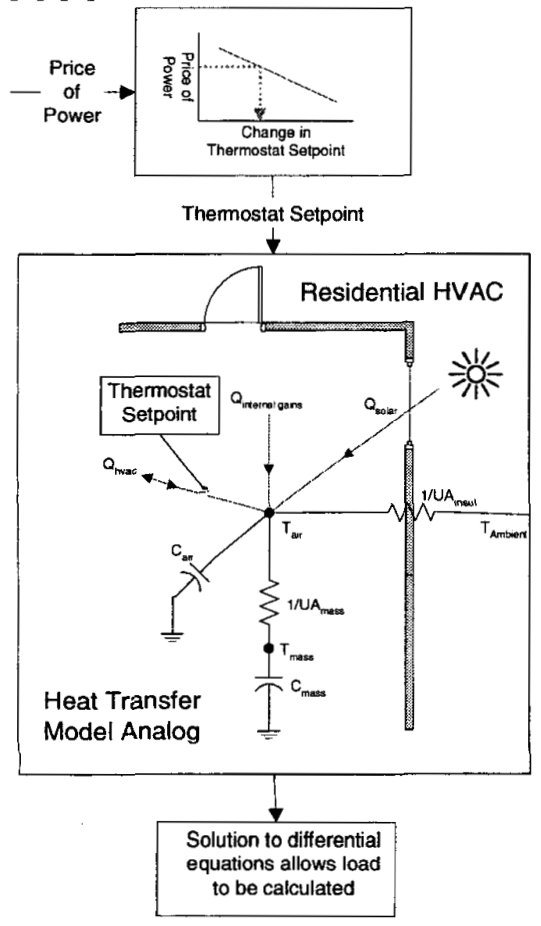

Fig. 4: Model of residential HVAC systems

The fundamental parameters in thermally controlled appliances are the size of the appliances, the nominal thermostat setpoint and ambient thermal conditions. Although each of these variables can be individually set for each appliance, the variables are typically set using random variables within bounds, assigned by uniform or Gaussian distributions.

The central part of Fig. 4 explains in detail how the thermally controlled appliances are modeled. Each type of appliance is modeled with a similar heat transfer model. However the constants within each model for each house are randomly distributed within expected ranges.

The rising curves in Fig. 5 indicate operation of the HVAC heater, and show a temperature rise within the house. The falling curve shown in Fig. 5 represents a cool down period where the home heating system is not active. As the HVAC unit cycles, the temperature in the house rises and falls with it. The cycling points of the HVAC unit are shown as times $\mathrm{t} 1$ - t4. These times indicate the end of a solution set, which is any event in the system such as reaching a temperature limit or reaching the end of a time step. At each end of a solution set, the coefficients of the differential equations are updated, then solved for time as a function of temperature.

This method of calculating load usage for thermostatically controlled loads has a tremendous advantage in computational speed over a finite difference method. Tradeoffs between accuracy and computation time can be easily made by changing the solution time step. Decreasing the time step enhances solution accuracy by allowing the differential equation coefficients to be updated more frequently.

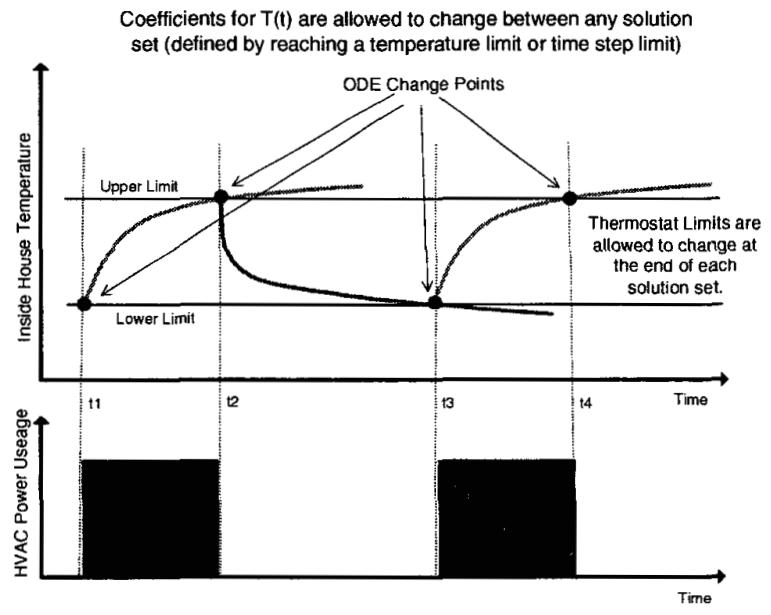

Fig. 5: Differential models of controlled thermal behavior

\section{B. Non-Thermostatically Controlled Appliances}

Appliances such as dishwashers, washers, and dryers cannot use the thermostatic method of curtailment because they are not controlled with a thermostat. A different technique of load deferral was designed based on a MonteCarlo method.

This approach, which computes the amount of load deferred for non-thermal loads, combines a Monte-Carlo method with a queuing technique. The calculation is analogous to a laundry system. Let the probability of doing a load of laundry be based on a probability density function (PDF) curve, as well as the amount of laundry in the laundry basket (which represents the queue). Let the queue increase by a linear function (in the absence of better information), and let it drop each time a load of laundry is done.

A form of Monte-Carlo method is used to determine if and when laundry will be done. A new curve is created called the 'Likelihood' curve. This function is the combination of the original PDF curve and the variable queue. The resulting PDF shape and amplitude represent the likelihood of doing a load of laundry on any particular day.

By comparing random numbers with the likelihood curve, a determination is made whether to do a load of laundry or not. Random numbers that fall beneath the curve represent a load of laundry being completed and a subsequent reduction of the 
queue, and those above the curve represent no laundry being done. Even though every laundry event is randomly assigned, a histogram of all laundry events for all homes approaches the shape of the original PDF curve as the number of homes increases.

Consider the implementation of a price responsive load using this Monte-Carlo process. If the likelihood graph in Fig. 6 represents the response to a residential spot price for electricity, then a curtailment strategy can be applied as follows. A curtailment curve is selected as a scaled and capped version of the spot market price (see Fig. 7, bottom graph). This signal is used to reduce the Likelihood curve, thus lowering the probability of an appliance usage event from occurring. This scheme results in the same number of events occurring on average, but the timing of events will be attracted to the lower priced hours, resulting in reduced consumer cost. For the scenario presented here, the cost per event was lowered by over $6 \%$.
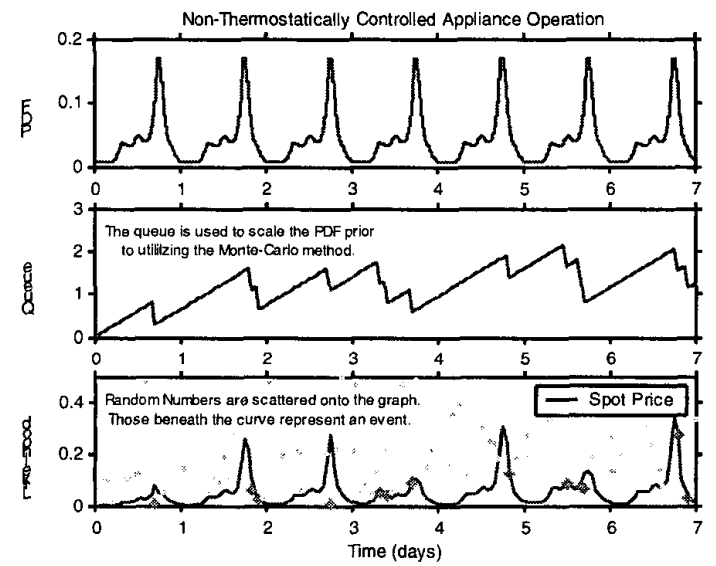

Fig. 6: Load shape generation

Precisely which curtailment strategy is selected is much less important than the ability to perform sensitivity studies by varying the related parameters.

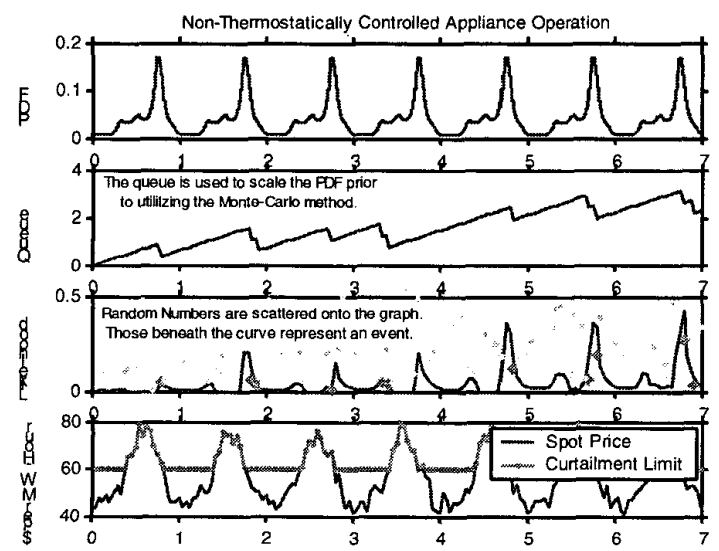

Fig. 7: Load shape generation with curtailment

\section{Human Behavior}

Human behavior can be approximated using a load-shape (i.e., diurnal and seasonal behaviors). The importance of economic decision-making behavior in the long-term evolution of power systems must also be considered.

The mechanism of contract selection is based on the modified Roth-Erev choice logic shown in Fig. 8. This method was chosen based principally on the analysis in [9]. Consumers receive a bill over an integrated period of time (for simplicity we assume this period is a month). Because consumers are different in their socio-economic characteristics (e.g., income, age, family size, region and climate, ownership) they have different expected utility functions. Expected utility functions reflect the deviation of the household consumption pattern from the average consumption as a result of differences in socio-economic characteristics drawn from the Consumer Expenditures Survey [10]. Consumers every month make a comparison between this month's bill and the expected electricity bill considering weather and past consumption. If the bill is less than the expected bill, the consumer will do nothing and wait until next month; otherwise, the consumer will assess the dollar value of the difference between the expected bill and the actual bill. If the difference is less than some critical value, the consumer will do nothing. If the difference exceeds a critical threshold of fitness, the consumer will explore the options for switching contracts and choose the option that would have yielded the lowest cost based on their history of consumption.

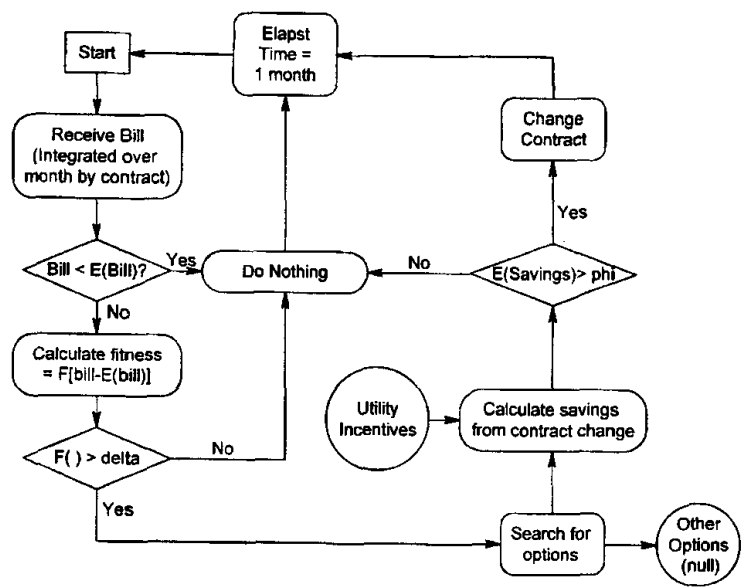

Fig. 8: Residential rate contract selection strategy

\section{EXOGENOUS INPUTS}

The residential appliances within a region show various levels of sensitivity to their ambient conditions. This exogenous information is configured as inputs to a residential feeder simulation system.

\section{A. Weather}

Weather has important impacts on the behavior of residential loads. The models accept weather data, which 
influences their behavior as they run in the simulation environment. Real, simulated, or even constant weather data can be easily used. The current implementation uses only temperature; however other weather parameters such as insolation, humidity, and wind can be added. Insolation affects the total heat gain, humidity primarily affects the cooling load, and wind affects the overall ventilation rate of the residence.

\section{B. System Voltage \& Frequency}

The voltage and frequency experienced at the head of the feeder is primarily driven by the system state at the transmission level. Voltage impacts are reflected in models of distribution system infrastructure and an associated power flow. The residential appliance models presently respond to nominal values of voltage and frequency, but are designed to be sensitive to changes in these values in the future. As an initial step, we plan to include the effect of voltage on induction motors and the effect of frequency on underfrequency controlled devices.

\section{Economic Conditions}

Wholesale power and fuel prices, as well as borrowing rates, all contribute parameters that drive the economic models used in the simulation. These economic models represent the contract choices to the consumer, which drive their behavior. Examples include fixed price, time of day, and spot market contracts.

\section{Time of Day, Day Type, Season}

The time of day, day type, and seasonal parameters are governed by a simulation clock that is advanced as the simulation proceeds in time. Every component of system has access to the clock and many use the clock to determine schedule-based changes in control and synchronization to playback tapes (e.g., weather, wholesale power prices, time of day rates).

\section{Aggregated, Feeder-Level Behavior}

To analyze the aggregated behavior of residential demand models, a distribution system simulation environment was constructed. Early experience with the simulator demonstrates complex load behavior that is not modeled in classical approaches.

\section{A. Simulation Environment}

A simulation environment packaged under the name Power Distribution System Simulation (PDSS) has been developed that makes use of the detailed residential demand models. A dialog-based interface provides users with access to the system model and various commands for controlling the simulator (see Fig. 9). Among other dialogs are a rate contract design tool, and an output selection tool (Fig. 10 and 11).

\section{B. Preliminary Simulation Experiences}

Initial runs of the simulator demonstrate behavior not supported by traditional load modeling methods. A prime example is the impact of abrupt rate changes on the diversity of load usually experienced at the head of a feeder.

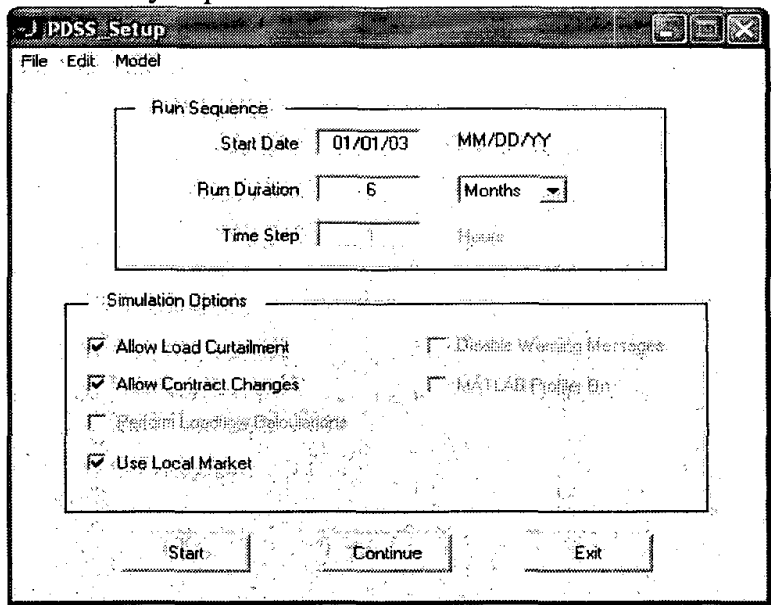

Fig. 9: PDSS main dialog

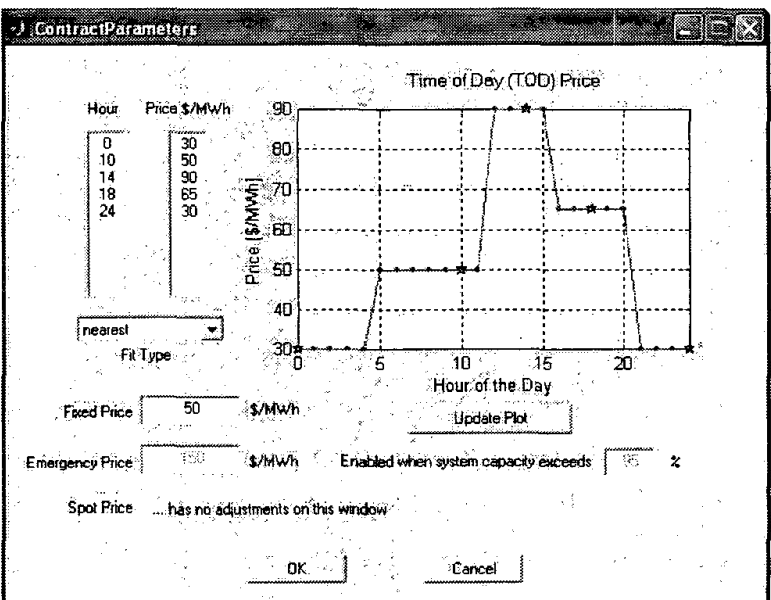

Fig.10: PDSS rate structure dialog

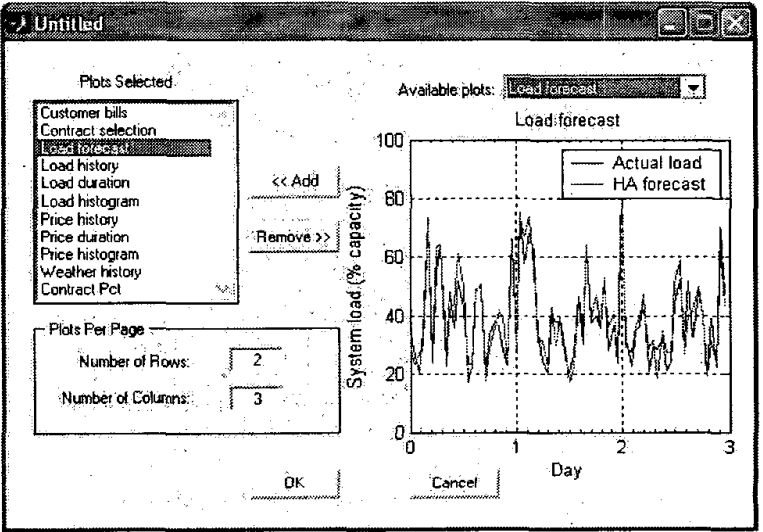

Fig.11: PDSS output selection dialog

Simulations of significant penetrations of customers on time of day or spot market contracts demonstrate that abrupt price increases cause many appliances to curtail load as expected; however, once the prices return to lower levels, the 
equipment resumes its consumption pattern. Much like the effect on resumption of service after a feeder outage, if all appliances on the feeder experience the same signal, then these economic signals can have the effect of synchronizing the demand patterns until load diversity is gradually restored over the course of a few hours. This can put unanticipated stress on the distribution system infrastructure.

In other simulations, time of day contracts were demonstrated to help "flatten" the load duration curve; however, preliminary indications from simple curtailment schemes reveal that peak conditions may not drop as dramatically as hoped. One reason appears to be the displacement of the peak to another time slot, instead of removing it all together.

\section{FUTURE WORK}

Further work is needed to calibrate these residential load models with other actual data sets besides the ELCAP data. Though each home's electric consumption is small relative to other building types, the number of different types of appliances is great. Models of commercial buildings are expected to be developed from the cornerstones laid down by the residential models. Similarly, large industrial consumers need to be modeled. Though some of the residential load modeling work may also apply to them, the custom nature of their equipment and configurations means that modeling such loads will likely require significant customization for each one.

To round out the distribution system simulator environment, the modeling must extend beyond load. Other areas for modeling include distributed generation and storage, more elaborate market economic models, simulated forecasts, as well as models for the communications systems that support information exchange and control. These models can benefit from work already being done by other researchers.

Finally, the complex interactive behavior that emerges on the distribution system will also have bulk transmission level impacts. Discovering reduced ordered equivalents of distribution feeders that adequately represent the behavior of load to changes in system conditions will become necessary for analyzing the implications of these feeders on the power grid. In addition, such equivalents can bring understanding to the influence of electrical and economic signal interactions between the transmission level and the distribution level of the system.

\section{REFERENCES}

[1] E. Hirst, "Reliability Benefits of Price-Responsive Demand," IEEE Power Engineering Review, vol. 22, no. 11, November 2002.

[2] S. W. Heunis and R. Herman, "A Probabilistic Model for Residential Consumer Loads," IEEE Transactions on Power Systems, vol. 17 no. 3, Aug. 2002.

[3] H.L. Willis, Power Distribution Planning Reference Book, Marcel Dekker Inc., 1997.

[4] F. J. Peterson, J. E. Patton, M. E. Miller, R. A. Gillman, W. M. Warwick and W. F. Sandusky, "End-Use Load and Consumer Assessment Program," Energy and Buildings, ISSN 0378-7788, vol. 19, no. 3, 1993
[5] R. G. Pratt, C. C. Conner, B. A. Cooke, and E. E. Richman, "Metered End-Use Consumption and Load Shapes from the ELCAP Residential Sample of Existing Homes in the Pacific Northwest," Energy and Buildings, ISSN 0378-7788, vol. 19, no. 3, 1993.

[6] R. G. Pratt, B. A. Ross, and W. F. Sandusky, "Analysis of Water Heater Standby Energy Consumption from ELCAP Homes," Energy and Buildings, ISSN 0378-7788, vol. 19, no. 3, 1993.

[7] R. G. Pratt and Z. T. Taylor, "Development and Testing of an Equivalent Thermal Parameter Model of Commercial Buildings from Time-Series End-Use Data," Pacific Northwest Laboratory, Richland, WA, Apr. 1994.

[8] Z. T. Taylor and R. G. Pratt, "The Effects of Model Simplifications on Equivalent Thermal Parameters Calculated from Hourly Building Performance Data," in Proceedings of the 1988 ACEEE Summer Study on Energy Efficiency in Buildings, Aug. 1988, pp 10.268-10.285.

[9] N. Nicolaisen, V. Petrov, L. Tesfastion, Market Power and Efficiency in a Computational Market with Discriminatory Double-action Pricing, ISU Economic Report No. 52, Aug. 27, 2000.

[10] "Consumer Expenditures Survey," Bureau of Labor Statistics, U.S. Department of Labor, http://stats.bls.gov/news.release/cesan.toc.htm.

\section{BIOGRAPHIES}

Ross T. Guttromson (M'01) received a BSEE degree from Washington State

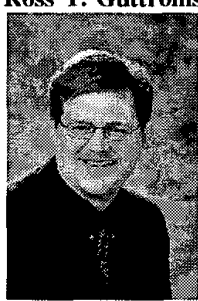
University, Pullman. Currently, he is a senior research engineer with the Energy Science \& Technology Division, Pacific Northwest National Laboratory, Richland, WA. He was with R. W. Beck Engineering and Consulting, Seattle, WA, from 1999 to 2001 and with the Generator Engineering Design Group, Siemens-Westinghouse Power Corporation, Orlando, FL, from 1995 to 1999 . Ross has one U.S. and one International patent, and is author and co-author of several papers on power systems and distributed resources. He is a member of the WECC Load Modeling Task Force, IEEE SCC $21 \quad$ P1547.2 standards group on Interconnecting Distributed Resources with Electric Power Systems, and the IEEE Wind Model Development Task Force. Ross is a U.S. Navy Submarine Veteran, having served on the USS Tautog (SSN 639) from 1987 to 1991.

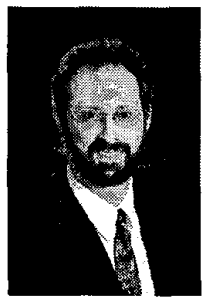

David P. Chassin received his BS of Building Science from Rensselaer Polytechnic Institiute in Troy, New York. $\mathrm{He}$ is staff scientist with the Energy Science \& Technology Division at Pacific Northwest National Laboratory were he has worked since 1992 . He was VicePresident of Development for Image Systems Technology from 1987 to 1992, where he pioneered a hybrid raster/vector computer aided design (CAD) technology called $\mathrm{CAD}$ Overlay $^{\mathrm{TM}}$. He has experience in the development of building energy simulation and diagnostic systems, leading the development of Softdesk Energy and DOE's Whole Building Diagnostician. He has served on the International Alliance for Interoperability's Technical Advisory Group, chaired the Codes and Standards Group, and served as the IAI Journal's editor from 1996 to 1998. His recent research focuses on emerging theories of complexity as they relate to highperformance simulation and modeling.

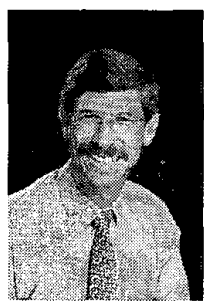

Steve Widergren (M'1978, SM'1992) received his BSEE (1975) and MSEE (1978) from the University of California, Berkeley. He works at the Pacific Northwest National Laboratory, where he contributes to the research and development of new solutions for the economic and reliable operation of power systems. Prior to joining PNNL, he was with ALSTOM ESCA, a bulk transmission energy management system supplier, where he designed software solutions for system operations and championed the establishment of an integrated suite of energy management system software products. He has also held power engineering positions at American Electric Power and Pacific Gas \& Electric (as an intern). $\mathrm{He}$ is vice-chair of the PES Energy Control Center Subcommittee and a member of the IEEE SCC 211547.3 standards group on monitoring, information exchange, and control of distributed resources. 